\title{
Targeting the MDM2-p53 interaction as a therapeutic strategy for the treatment of cancer
}

\section{Susan K Peirce Harry W Findley}

Department of Pediatrics, Division of Hematology and Oncology Emory University School of Medicine, Aflac Cancer Center and Blood Service, Atlanta, Georgia, USA
Correspondence: Susan K Peirce Department of Pediatrics, Division of Hematology and Oncology Emory University School of Medicine, Aflac Cancer Center and Blood Service, 2015 Uppergate Drive, Atlanta, Georgia 30322, USA

Tel + I 4047274766

Fax +I 404 727-4455

Email speirce@emory.edu
This article was published in the following Dove Press journal:

Cell Health and Cytoskeleton

24 June 2010

Number of times this article has been viewed
Abstract: The tumor suppressor p53 functions as an important defense against the development of cancer, and is negatively regulated by interaction with the oncogene and E3 ligase MDM2. In a tightly controlled system of feedback, MDM2 is, in turn, inhibited by the tumor suppressor $\mathrm{p} 14^{\mathrm{ARF}}$. The inhibition of MDM2-p53 interaction is an appealing therapeutic strategy for the treatment of cancer, and significant advances have been made in the development of small-molecule inhibitors which block this interaction and reactivate wild-type p53. However, the p53 gene is frequently mutated or deleted in cancer, or the wild-type $\mathrm{p} 53$ function inhibited by high levels of MDM2. Neuroblastoma (NB) is one such cancer and has presented a major therapeutic challenge in pediatric oncology. Although most NB tumors have wild-type p53, the p14 ${ }^{\mathrm{ARF}} / \mathrm{MDM} 2 / \mathrm{p} 53$ pathway is often altered, leading to resistance to many mainstay chemotherapeutics and a high incidence of relapse. In preclinical studies, the MDM2/p53 interaction inhibitor nutlin-3a has shown effectiveness in the treatment of chemoresistant NB with wild-type, mutant or null-p53 status, indicating that nutlin-3a has potential for the treatment of a broad range of chemoresistant and relapse tumors.

Keywords: p53, MDM2, MDMX, TAp73, nutlin-3a

\section{Introduction}

The p53 protein was first identified in $1979 .{ }^{1-3}$ It was initially thought to be an oncogene because of its antigenic association with SV40, a transforming tumor virus. ${ }^{4}$ Thus for a number of years it was not recognized as a key regulator of cell cycle arrest, DNA repair, senescence, autophagy and apoptosis. Its role as a tumor suppressor was not defined until 1989. ${ }^{5}$ The p53 gene, TP53, was cloned in $1983,{ }^{6}$ which led to the discovery that its mutation or deletion occurs in upwards of half of all cancers. In 1990, alteration of the gene was definitively linked to a rare familial syndrome, Li-Fraumeni, which is associated with various cancers including adrenocortical tumors, brain and breast cancers. $^{7}$ Tumor-associated p53 mutations are generally single base substitutions that lead to inhibition of normal function and sometimes confer new oncogenic properties (gain of function). ${ }^{8}$

As a transcription factor, p53 coordinates its network of actions primarily through its ability to transactivate target genes induced by the cellular stresses of DNA damage, ionizing radiation, UV exposure and a host of other internal and external events. Its levels are in large part regulated by the properties of the mouse double minute 2 protein, MDM2 (HDM2, human), an oncogene product and E3 ubiquitin ligase (an enzyme involved in coupling ubiquitin, a degradation tag, to lysine 
residues). ${ }^{9}$ MDM2 is transcriptionally upregulated by $\mathrm{p} 53$ following p53 activation. In turn, MDM2 acts to inhibit p53 action by a, binding to the transactivation region of $\mathrm{p} 53,{ }^{10} \mathrm{~b}$, promoting nuclear export of the p53 protein ${ }^{11}$ and c inducing ubiquitin-mediated proteosomal degradation. ${ }^{12}$ Thus MDM2 inhibits both the p53-mediated transactivating function of the p53 protein and the stability of the p53 protein itself. Such autoregulatory feedback tightly controls p53 levels to maintain homeostasis in unstressed cells and to restore homeostasis following stress responses. ${ }^{13}$

In roughly half of human cancers, the p53 gene is normal, but wild-type p53 function is inhibited by either inactivation of the cell cycle inhibitor and tumor suppressor $\mathrm{p} 14^{\mathrm{ARF}}$ or the amplification and overexpression of MDM2. ${ }^{14}$ MDM2 overexpression is linked to non-Hodgkin's lymphomas and B cell chronic lymphocytic leukemias, ${ }^{15}$ and a single nucleotide polymorphism at position 309 of intron 1 leads to several-fold increased levels of MDM2 in clusters of familial cancer. ${ }^{16}$ A second protein, MDMX (MDM4, mouse), a homologue of MDM2, is now known to stabilize the interaction between p53 and MDM2, thus promoting the oncogenic properties of MDM2. ${ }^{17}$

Both MDM2 and MDMX have been identified as potential therapeutic targets for treatment of cancer. Small molecule antagonists of MDM2 have been developed which disrupt the interaction between MDM2 and p53, leading to p53 stabilization and activation. ${ }^{18,19}$ The best known of these, nutlin-3a, is a nonpeptidic small-molecule inhibitor containing a cis-imidazoline core structure. It binds with high affinity to the N-terminal region of MDM2 in the p53 binding pocket. Importantly, it is nongenotoxic, in contrast to most chemotherapeutic compounds, a property that has led to its extensive preclinical study in models of human cancers. $^{20}$

Neuroblastoma (NB) is the most common extracranial solid tumor of childhood, and arises from the neural crest element of the sympathetic nervous system. ${ }^{21} \mathrm{NB}$ displays a wild-type p53 genotype at diagnosis and generally responds to initial therapy. However, relapse is common, in which the p53/ MDM2 pathway is deregulated, or, less commonly, in which p53 is directly mutated, resulting in chemoresistance..$^{22-24}$ Nutlin-3a is effective in the treatment of both chemosensitive and chemoresistant NB, including tumors with either wild-type or mutant $\mathrm{p} 53 .{ }^{25,26}$ In this review, we will discuss the key functional characteristics of p53 and MDM2, and the molecular mechanisms that underlie the effectiveness of MDM2-p53 targeted therapeutics for cancer, using NB as the model system.

\section{p53 structure, activation, and function}

p53 is a 393 amino acid nuclear phosphoprotein consisting of four functionally critical domains. The first 92 residues contain a transactivation (TA) and proline-rich domain, which is responsible for binding basal transcription factors including TFIID/TAF ${ }^{27}$ and which responds to DNA damage, UV or ionizing radiation. In response to such signals, Ser15 and Ser20 are rapidly phosphorylated. ${ }^{28}$ These phosphoryations are carried out by the protein kinases ATM/ATR (ataxia telangiectasia mutated and Rad3 associated), DNA-PK and Chk1/Chk2 (checkpoint kinase 1and 2), which function to mediate cell cycle checkpoints and DNA repair. ${ }^{29,30}$ The initial outcome, which occurs rapidly after stress, is to inhibit interaction between $\mathrm{p} 53$ and MDM2, inducing the immediate stabilization of p53; however, studies with knockin mouse models have shown that 553 stabilization in vivo also occurs in the absence of phosphorylation, ${ }^{31}$ implying a greater extent of complexity in the activation of $\mathrm{p} 53$.

The highly conserved core DNA binding domain (DNABD) lies between p53 residues 102 and 292. It is folded into a $\beta$ sheet containing short $\alpha$ helices, and contains two inverted pentameric sequences which act to bind to a consensus DNA sequence (response element, RE) in or near promoters of target genes. ${ }^{4,7}$ The $\mathrm{p} 53$ protein functions transcriptionally as a tetramer, and the residues 324-355 are necessary for this oligomerization..$^{32}$ The C-terminal basic domain (amino acids 356-393) contains a nuclear export signal as well as six lysines which contribute to ubiquitination by MDM2, leading to p53 proteasomal degradation. When acetylated by the histone acetyltransferase $\mathrm{CBP} / \mathrm{p} 300$ (CREB-binding protein) in response to stress, they are no longer targeted by MDM2, and the stability of p53 is increased. p53 stability is also increased by the binding and sequestration of MDM2 to the nucleolus by the tumor suppressor p14 ${ }^{\mathrm{ARF}}$. p14 ${ }^{\mathrm{ARF}}$ is present at low levels in unstressed cells, and its levels rise dramatically in response to stress signals. In addition to binding and sequestering MDM2, p14 ${ }^{\mathrm{ARF}}$ also inhibits MDM2 ligase activity. ${ }^{33}$

The effects of p53 stabilization and activation include cell cycle arrest, DNA repair, senescence, apoptosis and autophagy. Some of these effects may be mediated by transcriptionindependent means, but most are mediated directly by p 53 transactivation of target genes. ${ }^{34}$ Cell cycle and growth arrest result from $\mathrm{p} 53$ induction of $\mathrm{p} 21$ and Gadd45 (growth arrest and DNA-damage protein), and partial p53 acetylation is needed for this. Gadd45 functions as a stress sensor of genotoxic and other stresses; the Gadd45 protein interacts with $\mathrm{p} 21$ 
to halt $\mathrm{G} 1 / \mathrm{S}$ and $\mathrm{G} 2 / \mathrm{M}$ progression. ${ }^{35} \mathrm{p} 21$ is a cyclin-dependent kinase (CDK) inhibitor, and its continued transcription eventually leads to cell senescence. $\mathrm{p} 53$ promotes DNA repair by a transcription-dependent means through upregulation of proteins such as p53R2 (a p53-inducible small subunit of ribonucleotide reductase). It also acts more directly by a transcription-independent mechanism, by interaction with DNA repair proteins such as 53BP1 (a DNA double-strand break repair protein). ${ }^{36}$

The best known role for p53 is its transcriptional activation of genes involved in both intrinsic apoptosis (Bax, Puma, Noxa) and extrinsic apoptosis (Fas, DR4/DR5, TRAIL-R2). ${ }^{37}$ Intrinsic pathways induce signals initiated by DNA damage and other stresses, resulting in release of cytochrome c from the mitochondria, whereas extrinsic pathways induce apoptosis by the stimulation of death receptors located on the plasma membrane. Both pathways ultimately involve the activity of a group of cysteine proteases, the caspases, which share a common effector caspase, caspase 3 . In contrast to the activation of genes involved in cell cycle arrest and DNA repair, full p53 acetylation is required for the activation of these apoptotic genes; thus, the extent of p53 acetylation, and especially C-terminal acetylation, is a function of irreversible cellular damage. ${ }^{38}$ The importance of p53 acetylation is underscored by the presence of $\mathrm{CBP} / \mathrm{p} 300$ mutations in several cancers. Moreover, following stress responses, high cellular levels of deacetylases are induced to quickly disable p53 function. ${ }^{39}$

Bax, a key apoptotic protein, is a member of the large Bcl-2 family, members of which share four conserved domains, BH1-4 (Bcl-2 Homology 1-4). The Bcl-2 family contains both antiapoptotic and proapoptotic members. Bax is a direct initiator of cell death and is localized to the mitochondria where it homodimerizes and ultimately induces the release of cytochrome c. ${ }^{40}$ Puma and Noxa, in contrast, contain a single $\mathrm{BH} 3$ domain and function more indirectly by binding to and inhibiting prosurvival Bcl-2 family members. ${ }^{40}$ Fas, DR4/DR5 and TRAIL-R2 are members of the tumor necrosis factor (TNF) family, and function as either cell surface receptors or ligands in the induction of apoptosis. ${ }^{41}$

It is now known that the $\mathrm{p} 53$ protein can itself play a direct apoptotic role by binding to the mitochondrial membrane and Bcl-2 family members. p53 has been shown to localize to the mitochondria and interact with the prosurvival proteins $\mathrm{Bcl}-\mathrm{XL}$ and Bcl-2, allowing Bax and Bak (another proapoptotic member) to oligomerize and induce pore formation, releasing cytochrome c. p53 has also been shown to interact directly with Bak, freeing it from interaction with Mcl1, a third prosurvival member. ${ }^{42}$

\section{MDM2 structure and function}

The gene for MDM2 encodes a 489 amino acid protein, which includes 12 exons, two p53-response elements (RE) and two promoters. Transcription from these promoters generates two MDM2 proteins: the full-length and fully functional p90, and a p76 protein. This shorter protein lacks p53-binding ability and acts as a dominant negative inhibitor of $\mathrm{p} 90 .{ }^{43}$ Genetic studies in mice initially revealed that MDM2 is required as inhibitor of p53 function, as the embryonic lethality of MDM2-null mice is reversed by p53 knockout. ${ }^{44}$ To function as a negative regulator of p53 transcriptional activity, the N-terminus of the full-length MDM2 must bind to the $\mathrm{N}$-terminus of p53. The first approximately 120 amino acids of MDM2 contain a short, hydrophobic surface pocket and four amino acids in p53 mediate binding to this region: Phe19, Leu22, Trp23 and Leu26. ${ }^{45}$ MDMX (MDM4) also acts as a negative regulator of p53, again by inhibition of p53 transactivation and the promotion of MDM2 function, but it does not mediate p53 degradation. ${ }^{17}$ (Figure1)

Initially, it was thought that MDM2 interacted only with the p53 N-terminus. It is now known that MDM2 binds to both the DNABD and C-terminal domains of p53. Inhibition of this binding, in combination with interplay between posttranslational modifications such as phosphorylation and acetylation, releases p53 from repression. ${ }^{46}$ Interestingly, it has recently been shown that TAFII250 (a component of TFIID, a general transcription factor), has multiple functions in MDM2-mediated regulation of p53. It binds and stimulates the TA domain of p53; conversely, it also promotes MDM2-dependent turnover of p53 by downregulating MDM2 autoubiquitination and promoting p53-MDM2 interaction through interaction with the acidic domain of MDM2. Thus, while playing an essential role in basal p53 transcription, TAFII250 also stimulates p53 ubiquitination and degradation. ${ }^{47}$

The three major roles of MDM2 are: (1) the inhibition of p53 transactivation by binding to the TA region of $\mathrm{p} 53$; (2) the promotion of nuclear export and cytoplasmic accumulation of $\mathrm{p} 53$ by monoubiquitination and; (3) the induction of p53 proteosomal degradation by polyubiquitination of six key lysines in the p53 C-terminus. However, polyubiquitination of these lysines is not a universal requirement for MDM2-mediated degradation. In some cases, it requires other lysines, as shown by the substitution of these six lysines with arginine, or by their deletion. In such cases, MDM2 can 
still promote the degradation of $\mathrm{p} 53 .{ }^{48}$ Early work proposed that the C-terminal p53 domain functioned only as a target of regulation by MDM2. However, it has since become apparent that this domain interacts with the DNABD in the activation of p53-specific genes; acetylation of the C-terminal region positively affects the ability of p53 to bind DNA. The revised view is that the DNABD provides sequence-specificity, the C-terminal domain recognizes structural features of target DNA, and both are necessary for the activation of p53 target genes. ${ }^{38}$

Other MDM2-interacting proteins also regulate MDM2 function. The phosphorylation of MDM2 contributes to both the stabilization and inhibition of p53 function: phosphorylation of Tyr394 by c-Abl inhibits MDM2 ligase activity, ${ }^{49}$ whereas phosphorylation of Ser 166 and Ser 186 by Akt/PKB promote its E3-ligase activity. ${ }^{50}$ MDM2 mediates its own ubiquitination and degradation and is also ubiquitinated by other E3-ligases. MDM2 is deubiquitinated by Hausp, with which it forms a complex with Daxx (death-domain associated protein). Daxx stabilizes MDM2, and in a complex crosstalk of regulation, Daxx is deubiquitinated by Hausp, but ubiquitinated by MDM2. Thus, the interaction of MDM2 with Daxx may play a critical role in p53 activation. ${ }^{51,52}$

\section{Alternative model for $\mathrm{p} 53$ activation}

The traditional model of p53 transactivation was premised on a three-step process: The phosphorylation of key serines by ATM/ATR following a stress response, the stabilization and activation of p53 following release from MDM2, and the transcriptional targeting of response elements within or proximal to key genes by p53. A recently proposed alternative model suggests that p53 is, instead, constitutively and intrinsically activated. ${ }^{31}$ According to this model, activated p53 binds p21 and MDM2 genes in unstressed cells, but is repressed by the actions of MDMX and its homologue, MDM2. ${ }^{38,51}$ MDMX appears to play a more important role in this repression, as evidenced by the lethality of MDMX knockout mice containing functional MDM2. ${ }^{53}$ Thus, MDMX/MDM2 forms a complex with activated $\mathrm{p} 53$, which requires antirepression for transcriptional function. ${ }^{31}$ This alternate version for inducing p53 transcriptional activity suggests the following sequence of events: p53 is stabilized in response to stress via a number of mechanisms, many of which inhibit MDM2 and ubiquitination. This is followed by acetylation of p53 lysines, leading to antirepression and release of p53 from MDM2/MDMX and the phosphorylation of key serines on p53. Full activation necessitates the interaction of p53 with cofactors, some of which modify p53 as well as target histones and other transcriptional activators.

\section{Therapeutic targeting of MDM2-p53 interaction}

X-ray crystallography has been used to map the site of interaction between p53 and MDM2. This site has been identified as the N-terminus of p53 which contains four hydrophobic residues which interacts with a deep hydrophobic pocket within the first approximately 120 amino acids of MDM2. Three of these p53 amino acids are critical for this binding: Phe19, Trp23 and Leu26. ${ }^{9,54}$ Many experimental approaches have been used to demonstrate that the antagonism of MDM2 results in activation of $\mathrm{p} 53$ tumor suppression. These approaches include the inhibition of MDM2 expression, the inhibition of MDM2 ubiquitin ligase activity, and, most clinically relevant, the use of nonpeptidic small-molecule inhibitors to block interaction between MDM2 and p53. ${ }^{55,56}$ Beginning in the 1990s, studies of MDM2 downregulation

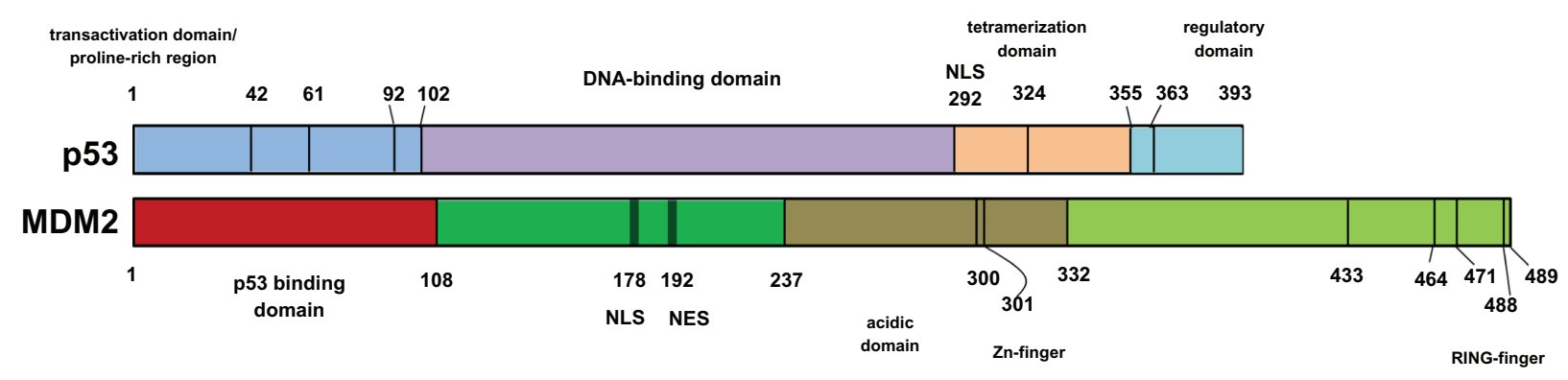

Figure I Schematic of the functional domains of p53 (top) and MDM2 (bottom). In unstressed cells, three N-terminal p53 residues (FI9, W23 and L26) are indispensible for binding to a small, deep hydrophobic pocket in the N-terminus of MDM2 to inhibit p53 function. The transactivation/proline-rich domain of $p 53$ responds to stress signals of DNA damage/radiation/UV by site-specific phosphorylation. The C-terminus ("regulatory domain") of p 53 contains six lysine residues which respond to stress by acetylation, inhibiting MDM2-mediated ubiquination, carried out within the RING-finger motif of MDM2. Zinc fingers generally act as motifs for protein-protein or protein-nucleic acid interactions, but the exact role in MDM2 is unclear. The acidic domain of MDM2 contains residues essential for phosphorylation and regulation, and residues $464-47$ I contain a nucleolar localization signal.

Abbreviations: NLS, nuclear localization signal; NES, nuclear export signal; Zn-finger, Zinc finger. 
using antisense oligonucleotides have demonstrated p53 activation. ${ }^{57,58}$ Although these antisense oligonucleotides suppressed tumor growth in mouse xenograft models, they have been difficult to develop for clinical applications. A second generation of MDM2 antagonists targeted the E3 ligase activity of MDM2 but lacked potency and specificity and demonstrated p53-independent off-target effects. ${ }^{59}$

More recently, the design of nonpeptidic, small-molecule MDM2 inhibitors has provided the proof-of-concept needed to advance the development of a number of small molecule inhibitors now in use in many preclinical studies. Small molecule MDM2 inhibitors were developed with three essential therapeutic properties in mind: 1) high binding specificity and affinity to MDM2, 2) potent anticancer efficacy especially in cancers with p53 wild-type (wt) status, and 3) favorable pharmacological and pharmacokinetic properties.

Several classes of these inhibitors have now been reported. These include analogs of spiro-oxindole, benzodiazepine, terphenyl, quilinol, chalone and sulfonamide, among others. Two of the most potent nonpeptidic MDM2 inhibitors (MI-63 and MI-219) are based on a spiro-oxindole structure, and one (MI-63) was found to have an extraordinarily high $\mathrm{K}$ of $3 \mathrm{~nm}$; however, MI-63 displayed unfavorable pharmacological properties in vivo in mouse studies. MI-219 (Ascenta Therapeutics, Malvern, PA, USA) was developed as an ana$\log$ of MI-63 to overcome these properties. ${ }^{19}$ Preliminary data in human cell lines have shown that MI-219 is a promising therapeutic for treatment of cancer with wild-type p53 and lacks toxicity to normal cells. ${ }^{60}$ Two benzodiazepines (BDPs) have been identified that disrupt the MDM2-p53 interaction, but in vitro studies are incomplete. ${ }^{19}$

The most widely reported of the MDM2 inhibitors are analogs of $c i s$-imidazoline, which includes nutlin-3a (Hoffman-La Roche, Nutley, NJ, USA). To date, 160+ preclinical cancer therapy studies using nutlin-3a have been published. The nutlins were isolated from a racemic mixture in 2004 by Vassilev et al. ${ }^{20,61}$ Nutlin-3a, an active enantiomer of nutlin-3, binds to MDM2 with high affinity $\left(\mathrm{K}_{\mathrm{i}}=36 \mathrm{~nm}\right)$. It blocks intracellular interaction of p53 with MDM2 by binding the N-terminal region of MDM2, inducing accumulation and activation of p53. In contrast to the vast majority of chemotherapeutics, nutlin-3a is nongenotoxic and does not typically induce apoptosis. Instead, it acts primarily by promoting cell cycle arrest, ${ }^{20,55,62}$ cell senescence ${ }^{63}$ and differentiation. ${ }^{64}$ The stabilization, activation or antirepression of $\mathrm{p} 53$ by nutlin-3a are sufficient to activate genes involved in blocking cell cycle progression. However, these effects are insufficient to induce apoptosis, which requires additional p53 posttranslational modifications. ${ }^{65}$ Thus, in preclinical trials, to promote apoptosis, nutlin-3a is usually used in combination with other chemotherapeutics. In tumors containing wt p53, it synergizes with the genotoxic agent doxorubicin, ${ }^{66}$ and it has also been shown to be effective in inducing proteins of the extrinsic apoptotic pathway, such as tumor necrosis factor-related apoptosis-inducing ligand (TRAIL). ${ }^{41}$ It has shown particular effectiveness when used in combination with drugs that target cell cycle progression, by inducing cell cycle arrest in G1-S and G2-M while protecting noncancerous cells. Pretreatment with nutlin-3a before treatment with taxanes, which kill cells in $\mathrm{M}$ phase, protects normal cells. Likewise, pretreatment before gemcitabine and Ara-C therapy (both of which kill cells in S-phase) block normal cells in G1 and G2, respectively. Thus, activation of p53 in the absence of DNA damage protects normal tissues. This lack of genotoxicity to normal tissues is important therapeutically, although the regulatory mechanisms are not fully understood. ${ }^{60,61}$ (Figure 2)

Both nutlin-3a and MI-219 bind to MDM2 with a much higher affinity than to MDMX. However, MDMX modulates the activity of MDM2 inhibitors; tumors which overexpress MDMX are known to abrogate the action of MDM2 inhibitors, and cell lines with lower MDMX levels are more sensitive to nutlin-3a treatment. ${ }^{53}$ Similarly, the tumors most responsive to MDM2 inhibitors are those overexpressing MDM2 with low MDMX expression. These findings suggest that small molecule inhibitors should be developed that target both MDM2 and MDMX.

The retinoblastoma protein $(\mathrm{Rb})$ is a tumor suppressor protein inactivated in many cancers. One of its best known functions is cell-cycle regulation. One recent insight into nutlin-3a action comes from studies of the $\mathrm{Rb}$ tumor suppressor. ${ }^{67}$ p53 is required for transcription of the $\mathrm{Rb}$ gene, ${ }^{68}$ and the $\mathrm{Rb}$ protein interacts with E2F1 (a member of the E2F transcription factor family with apoptotic properties) to induce assembly of transcription repressor functions $;{ }^{69,70}$ these inhibit expression of E2F target genes usually involved in cell cycle progression. In this manner, $\mathrm{Rb}$ represses cell cycle progression genes. Hypophosphorylation of Rb leads to suppression of constitutive growth, ${ }^{71}$ and p21 is responsible for accumulation of hypophosphorylated $\mathrm{Rb}$. In most cells, treatment with nutlin-3a induces both increased levels of Rb hypophosphorylation and cell cycle arrest, while in a small subset of cells, lower levels of hypophosphorylated $\mathrm{Rb}$ are induced and cells enter apoptosis. ${ }^{72}$ Thus, it appears that high levels of hypophosphorylated $\mathrm{Rb}$ inhibit apoptosis 


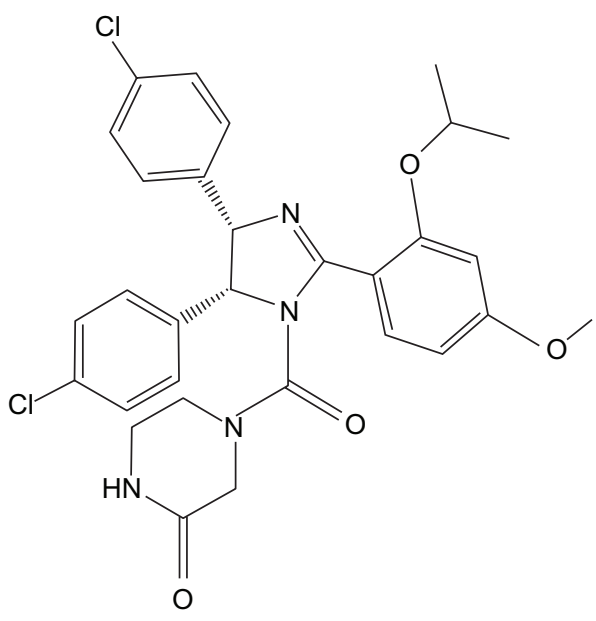

(a) Nutlin-3a

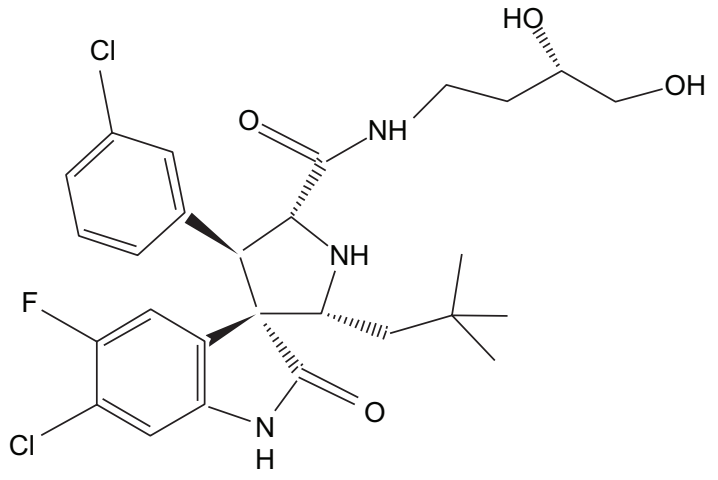

Figure 2 Chemical structures of nutlin-3a (a) and MI-219 (b), two small-molecule inhibitors of the MDM2-p53 interaction in advanced preclinical development for the treatment of cancer. Nutlin-3a is a cis-imidazoline analog, and MI-219 is a spiro-oxindole analog.

induced by nutlin-3a, and instead promote cell-cycle arrest, effects that are context-dependent. ${ }^{67}$

E2F1 plays an important role in the response of cells and tumor tissues to nutlin-3a treatment. E2F1 binds to MDM2 and nutlin-3a potentiates chemotherapy by inhibiting this interaction and inducing overexpression of E2F1. Overexpression of E2F1 is known to increase Bax translocation from the cytosol to the mitochondria, ${ }^{73}$ and expression of the apoptotic protein Puma is transcriptionally upregulated by E2F1, which contains six E2F1 binding sites in its promoter.

Nutlin-3a has an inhibitory effect on angiogenesis. Hypoxia, a typical feature of advanced tumor growth, promotes angiogenesis by upregulating vascular endothelial growth factor (VEGF), a key angiogenic factor. Under hypoxic conditions, HIF-1alpha and its transcriptional target VEGF are both upregulated. An earlier study indicated that the inhibition of MDM2 interaction with HIF-1alpha promotes its degradation, leading to suppression of VEGF expression. ${ }^{75}$ Recently, it was shown that nutlin-3a directly inactivates the $\mathrm{C}$-terminal transactivation domain of HIF-1alpha, leading to an inhibition of the expression of VEGF. ${ }^{74}$

\section{Nutlin-3 is effective in the absence of functional p53}

p53 is mutated or absent in many cancers, a feature which has major therapeutic implications. p53 is a member of a large family of proteins of which p63 is the ancestral gene. From genetic analyses, it has been found that p73 and then p53 evolved from this ancestral form. ${ }^{76}$ Unlike p53, p73 family members are rarely mutated in cancer. TAp73 (transactivating $\mathrm{p} 73$, functionally similar to $\mathrm{p} 53$ ) and $\Delta \mathrm{Np} 73$ (TA-truncated $\mathrm{p} 73$ ) act with p53 to maintain cellular equilibrium. $\Delta \mathrm{Np} 73$ occurs as one of 4 isoforms, all of which act in a dominantnegative manner to suppress p53 and TAp73, while TAp73 can be induced by chemotherapeutic treatment (including camptothecin, etoposide, cisplatinum, doxorubicin, and Taxol) at low concentrations in cells with mutant or deleted p53. ${ }^{77}$ TAp73 is an important determinant of chemosensitivity in many human cancers; however, mutant p53 often contains polymorphisms that function to inhibit TAp73, thus inducing drug resistance. ${ }^{77,78}$ Mutant p53 and $\Delta \mathrm{Np} 73$ are able to inhibit p53 and TAp73 by direct neutralization of TAp73 function, heterocomplex formation and possibly by promoter competition. ${ }^{78,79}$ Many human cancers express upregulated $\Delta \mathrm{Np} 73$, and, even in the presence of wild-type $\mathrm{p} 53$, drug resistance may be present. ${ }^{80} \Delta \mathrm{Np} 73$ is also known to cooperate with the oncogenic Ras protein to induce high levels of phosphorylated $\mathrm{Rb},{ }^{81}$ and $\Delta \mathrm{Np} 73$ expression has a role in neuroblastoma oncogenicity. ${ }^{82}$

In model systems, nutlin-3 is effective in the absence of wild-type p53, a feature attributable to the presence of TAp $73 .^{83,84}$ In addition to its interaction with p53, the N-terminus of MDM2 interacts with TAp73 as well as E2F1. The three key p53 amino acids (Phe 19, Trp23 and Leu26) that bind MDM2 also function to bind TAp73 to MDM2. ${ }^{85}$ Thus, nutlin-3a treatment upregulates TAp73 function in the same manner in which it upregulates p53: by disrupting the interaction between MDM2 and TAp73, leading to TAp73 
stabilization. However, markedly higher concentrations of nutlin-3a are required for TAp73-dependent effects and E2F1 mediated apoptosis. ${ }^{86}$ Recently, cycloxygenase (COX) inhibitors have been shown to downregulate the multiple isoforms of $\triangle \mathrm{Np} 73$ in $\mathrm{NB} ;{ }^{85}$ combination treatment of $\mathrm{COX}$ inhibitors with nutlin-3a may represent a novel approach to the treatment of NB containing high levels of $\Delta \mathrm{Np} 73$ in the absence of wild-type p53.

\section{MDM2 inhibition for treatment of neuroblastoma}

Neuroblastoma (NB) is a tumor derived from precursor cells of the sympathetic nervous system and the second most common solid tumor in children. Mutations of p53 are absent at diagnosis, although wild-type p53 is frequently hyperubiquitinated and sequestered in the cytoplasm. ${ }^{87}$ Most neuroblastomas respond to initial treatment, and complete regression without treatment can occur in infancy. Doxorubicin is a common first-line treatment. In addition to its role as an intercalator of DNA, it also functions as an inhibitor of MDM2 enzymatic activity, blocking MDM2 E3 ligase activity and hence the ubiquitination of p53. ${ }^{88}$ However, relapse with chemoresistant disease is common and is often associated with MDM2 overexpression, suppressed p14 ${ }^{\mathrm{ARF}}$ function, and less commonly, p53 mutations. ${ }^{22,23}$ High-risk NB remains one of the most problematic childhood cancers, and children with high-risk disease have a long-term survival rate of around $30 \% .^{21}$ The specific targeting of the $\mathrm{p} 14^{\mathrm{ARF}} / \mathrm{MDM} 2 / \mathrm{p} 53$ axis in NB has become a research area of keen interest.

$M y c N$ oncogene amplification occurs in approximately $25 \%$ of neuroblastomas and is a highly significant prognostic marker for poor survival, with many relapse cases of NB expressing the $\mathrm{MycN}$ protein at high levels. $\mathrm{MycN}$ is a member of the basic-helix-loop-helix zipper family of transcription factors and binds to an E-box motif consensus sequence within the promoter of target genes, including, most importantly, MDM2. Thus, $M y c N$ amplification and overexpression is linked to MDM2 overexpression. ${ }^{89,90} \mathrm{MycN}$ has also been shown to mediate centrosome amplification in NB, leading to karyotypic instability. ${ }^{91}$

Recent work provides evidence that, paradoxically, p53 is directly targeted for transcription by $\mathrm{MycN}$, which binds to an E-box motif within the p53 promoter. In these and other studies, fully functional p53 displays nuclear accumulation in neuroblastomas expressing high levels of $\mathrm{MycN}$. Because neuroblastoma derives from undifferentiated neuroblasts, it is conjectured that $\mathrm{MycN}$ and $\mathrm{p} 53$ function together in balance during early embryonic development, as $\mathrm{MycN}$ plays a role in p53-driven apoptosis. However, both $\mathrm{MycN}$ and p53 are frequently overexpressed in primary neuroblastomas. Studies of a MycN-transgenic mouse model have shown that targeted expression of $\mathrm{MycN}$ in neural crest lineage cells leads to hyperproliferation. ${ }^{12}$ MycN-overexpressing cells develop mechanisms for evading p53-induced apoptosis; this MycN phenotype is generally associated with an aberrant p14 ${ }^{\mathrm{ARF}} / \mathrm{MDM} 2 / \mathrm{p} 53$ pathway. ${ }^{92}$ For example, MycN-amplified and overexpressing neuroblastomas are often resistant to first line drugs (including doxorubicin, cyclophosphamide, vincristine, cisplatin and etoposide, among others) for treatment of NB. Therapy-resistant and relapse tumors also often display alterations including MDM2 overexpression and deletion or methylation of $\mathrm{p} 14^{\mathrm{ARF}}$, as well as downstream p53 mutations. ${ }^{23}$

In experimental settings, treatment with nutlin-3a results in rapid and dramatic accumulation of p53 in wt-p53 NB cells one to two hours following combination treatment with cisplatin or etoposide. ${ }^{26,93}$ This response to nutlin-3a response is independent of $M y c N$ amplification status. Recently, nutlin-3a treatment in combination with vincristine was found to be highly effective against p53-mutated multidrug resistant NB cells in vitro. ${ }^{94}$ Very recently, it was found that nutlin-3a is effective against chemoresistant NB containing both $\Delta \mathrm{Np} 73$ and wt-p53, and is able to suppress NB cell growth in a subcutaneous xenograft model system. ${ }^{95}$ In some NB cell lines, nutlin-3a promotes a senescence-like phenotype, while in others, it drives differentiation. ${ }^{95}$ In culture, endogenous TAp73 levels, but not p53 levels, induce differentiation of NB cells by both neurite outgrowth and by the downregulation of N-Myc and upregulation of $\mathrm{Rb} .{ }^{96}$ The therapeutic upregulation of TAp73 in NB may therefore be of clinical importance.

In our laboratory, nutlin-3a was used to sensitize a p53null and doxorubicin-resistant NB cell line to doxorubicin. We found that nutlin-3a treatment upregulated TAp73 and E2F1 protein levels, while also potentiating the ability of doxorubicin to block cell proliferation and activate apoptosis. ${ }^{84}$ Based on our results, we postulated a mechanism for the interaction of TAp73 and E2F1 in p53-null NB cells treated with combined nutlin-3a and doxorubicin. In untreated cells, TAp73 and E2F1 are bound to MDM2. Combination treatment leads both to nutlin-3a mediated dislocation of E2F1/TAp73 from MDM2 and doxorubicin-mediated DNA damage. Without TAp73 dislocation from MDM2, induction of downstream apoptotic proteins will occur at only low levels. DNA damage induces the checkpoint kinases Chk1/Chk2 
which subsequently phosphorylate both E2F1 and TAp73, stabilize E2F1 and promote TAp73 transcription. Thus, without concurrent DNA damage, we hypothesize that p53-null tumors will be resistant to nutlin-3a.

\section{Conclusions}

Activation of the p53 tumor suppressor pathway by MDM2 inhibition has been studied as a therapeutic strategy for the treatment of malignancies for over a decade. Although considerable progress has been made in the development of p53-MDM2 interaction inhibitors for the treatment of cancer, few have demonstrated the necessary specificity, potency or pharmacokinetic properties for in vivo evaluation. Nutlin-3a, a cis-imidazoline, is the most clinically promising and is currently in advanced preclinical development for treatment of tumors with wild-type p53. However, much less is known about the mechanisms of nutlin-3a action in systems where p53 is directly mutated or its function is inhibited. This would include chemoresistant cancers such as NB in which MDM2 is frequently overexpressed or the $\mathrm{p} 14^{\mathrm{ARF}} / \mathrm{p} 53$ pathway is altered. Recent data showing that nutlin-3a is inhibitory against p53-mutant and p53-null NB cells suggests that this agent may have a wide spectrum of activity in tumors regardless of their p53 status. This attribute, in combination with its low toxicity, should provide impetus for clinical trials of nutlin-3a in NB and other tumors characterized by an aberrant $\mathrm{p} 53 / \mathrm{MDM} 2 / \mathrm{p} 14^{\mathrm{ARF}}$ pathway.

\section{Acknowledgments}

We gratefully acknowledge helpful discussions with Dr. David Freedman, as well as editorial assistance.

\section{Disclosure}

The authors report no conflicts of interest in this work.

\section{References}

1. DeLeo AB, Jay G, Appella E, Dubois GC, Law LW, Old LJ. Detection of a transformation-related antigen in chemically induced sarcomas and other transformed cells of the mouse. Proc Natl Acad Sci U S A. 1979;76(5):2420-2424.

2. Lane DP, Crawford LV. T antigen is bound to a host protein in SY40transformed cells. Nature. 1979;278(5701):261-263.

3. Linzer DIH, Levine AJ. Characterization of a $54 \mathrm{~K}$ Dalton cellular SV40 tumor antigen present in SV40-transformed cells and uninfected embryonal carcinoma cells. Cell. 1979;17(1):43-52.

4. Levine AJ, Finlay CA, Hinds PW. p53 is a tumor suppressor gene. Cell. 2004;116(S 2):S67-S70.

5. Finlay CA, Hinds PW, Levine AJ. The $\mathrm{p} 53$ proto-oncogene can act as a suppressor of transformation. Cell. 1989;57(7):1083-1093.

6. Oren M, Levine AJ. Molecular cloning of a cDNA specific for the murine p53 cellular tumor antigen. Proc Natil Acad Sci U S A. 1983;80(1): $56-59$.
7. Vogelstein B, Lane D, Levine AJ. Surfing the p53 network. Nature. 2000;408(6810):307-310.

8. Whibley C, Pharoah PDP, Hollstein M. p53 polymorphisms: cancer implications. Nat Rev Cancer. 2009;9(2):95-107.

9. Iwakuma T, Lozano G. MDM2, An Introduction. Mol Cancer Res. 2003;1(14):993-1000.

10. Momand J, Zambetti GP, Olson DC, George D, Levine AJ. The MDM-2 oncogene product forms a complex with the p53 protein and inhibits p53-mediated transactivation. Cell. 1992;69(7):1237-1245.

11. Freedman DA, Wu L, Levine AJ. Functions of the MDM2 oncoprotein. Cell Mol Life Sci. 1999;55(1):96-107.

12. Haupt Y, Maya R, Kazaz A, Oren M. MDM2 promotes the rapid degradation of p53. Nature. 1997;387(6630):296-299.

13. Wu X, Bayle JH, Olson D, Levine AJ. The p53-MDM-2 autoregulatory feedback loop. Genes Dev. 1993;7(7a):1126-1132.

14. Polager S, Ginsberg D. p53 and E2f: partners in life and death. Nat Rev Cancer. 2009;9(10):738-748.

15. Watanabe T, Ichikawa A, Saito H, Hotta T. Overexpression of the MDM2 Oncogene in Leukemia and Lymphoma. Leuk Lymphoma. 1996;21(5):391-397.

16. Bond GL, Levine AJ. A single nucleotide polymorphism in the p53 pathway interacts with gender, environmental stresses and tumor genetics to influence cancer in humans. Oncogene. 2007;26(9): $1317-1323$.

17. Linares LK, Hengstermann A, Ciechanover A, Muller S, Scheffner M. HdmX stimulates HDM2-mediated ubiquitination and degradation of p53. Proc Natl Acad Sci U S A. 2003;100(21): 12009-12014.

18. Bianco R, Ciardiello F, Tortora G. Chemosensitization by antisense oligonucleotides targeting MDM2. Curr Cancer Drug Targets. 2005;5(1):51-56.

19. Shangary S, Wang S. Targeting the MDM2-p53 interaction for cancer therapy. Clin Cancer Res. 2008;14(17):5318-5324.

20. Vassilev LT. MDM2 inhibitors for cancer therapy. Trends Mol Med. 2007;13(1):23-31.

21. Brodeur GM. Neuroblastoma: biological insights into a clinical enigma. Nat Rev Cancer. 2003;3(3):203-216.

22. Carr J, Bell E, Pearson ADJ, et al. Increased frequency of aberrations in the p53/MDM2/p14ARF pathway in neuroblastoma cell lines established at relapse. Cancer Res. 2006;66(4):2138-2145.

23. Carr-Wilkinson J, O'Toole K, Wood KM, et al. High frequency of p53/MDM2/p14ARF pathway abnormalities in relapsed neuroblastoma. Clinl Cancer Res. 2010;16(4):1108-1118.

24. Tweddle D, Pearson A, Haber M, et al. The p53 pathway and its inactivation in neuroblastoma. Cancer Lett. 2003;197(1-2):93-98.

25. Kim E, Shohet J. Targeted molecular therapy for neuroblastoma: The ARF/MDM2/p53 axis. J Natl Cancer Inst. 2009;101(22): 1527-1529.

26. Barbieri E, Mehta P, Chen Z, et al. MDM2 inhibition sensitizes neuroblastoma to chemotherapy-induced apoptotic cell death. Mol Cancer Ther. 2006;5(9):2358-2365.

27. Levine AJ. p53, the cellular gatekeeper for growth and division. Cell. 1997;88(3):323-331.

28. Shimizu H, Hupp TR. Intrasteric regulation of MDM2. Trends Biochem Sci. 2003;28(7):346-349.

29. Banin S, Moyal L, Shieh S-Y, et al. Enhanced phosphorylation of p53 by ATM in response to DNA damage. Science. 1998;281(5383): 1674-1677.

30. Gonzalez S, Prives C, Cordon-Cardo C. p73\{alpha\} Regulation by Chk1 in response to DNA damage. Mol Cell Biol. 2003;23(22): $8161-8171$.

31. Kruse J-P, Gu W. Modes of p53 regulation. Cell. 2009;137(4): 609-622.

32. Liang S-H, Clarke MF. Regulation of p53 localization. Eur J Biochem. 2001;268(10):2779-2783.

33. Sherr CJ. Divorcing ARF and p53: an unsettled case. Nat Rev Cancer. 2006;6(9):663-673. 
34. Uo T, Kinoshita Y, Morrison RS. Apoptotic actions of p53 require transcriptional activation of PUMA and do not involve a direct mitochondrial/cytoplasmic site of action in postnatal cortical neurons. J Neurosci. 2007;27(45):12198-12210.

35. Taylor W, Stark G. Regulation of the G2/M transition by p53. Oncogene. 2001;20(15):1803-1815.

36. Brummelkamp T, Fabius A, Mullenders J, et al. An shRNA barcode screen provides insight into cancer cell vulnerability. Nat Chem Biol. 2006;2:202-206.

37. Vincenzo L, Gerry M. Evolution of functions within the $\mathrm{p} 53 / \mathrm{p} 63 / \mathrm{p} 73$ Family. Ann N Y Acad Sci. 2000;926: 90-100.

38. Tang Y, Zhao W, Chen Y, Zhao Y, Gu1 W. Acetylation is indispensable for p53 activation. Cell. 2008;133(4):612-626.

39. Costanzo A, Merlo P, Pediconi N, et al. DNA damage-dependent acetylation of $\mathrm{p} 73$ dictates the selective activation of apoptotic target genes. Molecular Cell. 2002;9(1):175-186.

40. Ramadan S, Terrinoni A, Catani MV, et al. p73 induces apoptosis by different mechanisms. Biochem Biophys Res Commun. 2005;331(3) 713-717

41. Hori T, Kondo T, Kanamori M, et al. Nutlin-3 enhances tumor necrosis factor-related apoptosis-inducing ligand (TRAIL)-induced apoptosis through up-regulation of death receptor 5 (DR5) in human sarcoma HOS cells and human colon cancer HCT116 cells. Cancer Lett 2010;287(1):98-108.

42. Marchenko N, Moll U. The role of ubiquitination in the direct mitochondrial death program of p53. Cell Cycle. 2007;6:1718-1723.

43. Evans SC, Viswanathan M, Grier JD, Narayana M, El-Naggar AK, Lozano G. An alternatively spliced HDM2 product increases p53 activity by inhibiting HDM2. Oncogene. 2001;20(30):4041.

44. Jones SN, Hancock AR, Vogel H, Donehower LA, Bradley A. Overexpression of $\mathrm{Mdm} 2$ in mice reveals a p53-independent role for Mdm2 in tumorigenesis. Proc Natl Acad Sci U S A. D1998;95(26): $15608-15612$.

45. Kussie PH, Gorina S, Marechal V, et al. Structure of the MDM2 Oncoprotein Bound to the p53 Tumor Suppressor Transactivation Domain. Science. 1996;274(5289):948-953.

46. Chan WM, Mak MC, Fung TK, Lau A, Siu WY, Poon RYC. Ubiquitination of p53 at multiple sites in the DNA-binding domain. Mol Cancer Res. 2006;4(1):15-25.

47. Allende-Vega N, Saville MK, Meek DW. Transcription factor TAFII250 promotes Mdm2-dependent turnover of p53. Oncogene. 2007;26(29):4234-4242.

48. Feng J, Tamaskovic R, Yang Z, et al. Stabilization of Mdm2 via decreased ubiquitination is mediated by protein kinase $\mathrm{B} / \mathrm{Akt}$-dependent phosphorylation. J Biol Chem. 2004;279(34):35510-35517.

49. Goldberg Z, Sionov RV, Berger M, et al. Tyrosine phosphorylation of $\mathrm{Mdm} 2$ by c-Abl: implications for p53 regulation. EMBO J. 2002;21(14):3715-3727.

50. Mayo LD, Seo YR, Jackson MW, et al. Phosphorylation of human p53 at Serine 46 determines promoter selection and whether apoptosis is attenuated or amplified. J Biol Chem. 2005;280(28):25953-25959.

51. Tang J, Qu L, Pang M, Yang X. Daxx is reciprocally regulated by Mdm2 and Hausp. Biochem Biophys Resh Commun. 2010;393(3):542-545.

52. Meek DW, Knippschild U. Posttranslational modification of MDM2. Mol Cancer Res. 2003;1(14):1017-1026.

53. Migliorini D, Lazzerini DE, Danovi D, et al. Mdm4 (Mdmx) regulates p53-induced growth arrest and neuronal cell death during early embryonic mouse development. Mol Cell Biol. 2002;22(15):5527-5538.

54. Bond GL, Hu W, Levine AJ. MDM2 is a central node in the p53 pathway: 12 years and counting. Curr Cancer Drug Targets. 2005;5(1):3-8

55. Carvajal D, Tovar C, Yang H, Vu BT, Heimbrook DC, Vassilev LT. Activation of $\mathrm{p} 53$ by MDM2 antagonists can protect proliferating cells from mitotic inhibitors. Cancer Res. 2005;65(5):1918-1924.

56. Hu B, Gilkes DM, Chen J. Efficient p53 Activation and apoptosis by simultaneous disruption of binding to MDM2 and MDMX. Cancer Res. 2007;67(18):8810-8817.
57. Wang H, Nan L, Yu D, Agrawal S, Zhang R. Antisense anti-MDM2 oligonucleotides as a novel therapeutic approach to human breast cancer: in vitro and in vivo activities and mechanisms. Clin Cancer Res. 2001;7(11):3613-3624.

58. Zhang R, Wang H, Agrawal S. Novel antisense anti-MDM2 mixed-backbone oligonucleotides: proof of principle, in vitro and in vivo activities, and mechanisms. Curr Cancer Drug Targets. 2005;5(1): 43-49.

59. Buolamwini JK, Addo J, Kamath S, Patil S, Mason M, Ores M. Small molecule antagonists of the MDM2 oncoprotein as anticancer agents. Curr Cancer Drug Targets. 2005;5(1):57-68.

60. Shangary S, Qin D, McEachern D, et al. Temporal activation of p53 by a specific MDM2 inhibitor is selectively toxic to tumors and leads to complete tumor growth inhibition. Proc Natl Acad Sci US A. 2008; 105(10):3933-3938.

61. Vassilev LT, Vu BT, Craves B, et al. In vivo activation of the p53 pathway by small-molecule antagonists of MDM2. Science. 2004;303(5659):844-848.

62. Tovar C, Rosinski J, Filipovic Z, et al. Small-molecule MDM2 antagonists reveal aberrant p53 signaling in cancer: Implications for therapy. Proc Natl Acad Sci U S A. 2006;103(6):1888-1893.

63. Kumamoto K, Spillare EA, Fujita K, et al. Nutlin-3a activates p53 to both down-regulate inhibitor of growth 2 and up-regulate mir-34a, mir-34b, and mir-34c expression, and induce senescence. Cancer Res 2008;68(9):3193-3203.

64. Maimets T, Neganova I, Armstrong L, Lako M. Activation of p53 by nutlin leads to rapid differentiation of human embryonic stem cells. Oncogene. 2008;27(40):5277-5287.

65. Elisa B, Federica C, Erika R, et al. Differential effects of chemotherapeutic drugs versus the MDM-2 antagonist nutlin-3 on cell cycle progression and induction of apoptosis in SKW6.4 lymphoblastoid B-cells. J Cell Biochem. 2008;104(2):595-605.

66. Drakos E, Thomaides A, Medeiros LJ, et al. Inhibition of p53-murine double minute 2 interaction by nutlin-3a stabilizes p 53 and induces cell cycle arrest and apoptosis in Hodgkin lymphoma. Clin Cancer Res. 2007;13(11):3380-3387.

67. Du W, Wu J, Walsh EM, Zhang Y, Chen CY, Xiao Z-XJ. Nutlin-3 affects expression and function of retinoblastoma protein. $\mathrm{J}$ Biol Chem. 2009;284(39):26315-26321.

68. Cam H, Griesmann H, Beitzinger M, et al. p53 family members in myogenic differentiation and rhabdomyosarcoma development. Cancer Cell. 2006;10(4):281-293.

69. Stevaux O, Dyson NJ. A revised picture of the E2F transcriptional network and RB function. Curr Opin Cell Biol. 2002;14(6):684-691.

70. La Thangue NB. The yin and yang of E2F-1: balancing life and death. Nat Cell Biol. 2003;5(7):587-589.

71. Knudsen ES, Wang JY. Dual mechanisms for the inhibition of E2F binding to RB by cyclin-dependent kinase-mediated RB phosphorylation. Mol Cell Biol. 1997;17(10):5771-5783.

72. Kitagawa M, Aonuma M, Lee SH, Fukutake S, McCormick F. E2F-1 transcriptional activity is a critical determinant of Mdm2 antagonistinduced apoptosis in human tumor cell lines. Oncogene. 2008;27: 5305-5314.

73. Hao H, Dong Y, Bowling M, Gomez-Gutierrez J, Zhou HS, McMasters K. E2F-1 induces melanoma cell apoptosis via PUMA up-regulation and Bax translocation. BMC Cancer. 2007;7(1):24.

74. Lee Y-M, Lim J-H, Chun Y-S, et al. nutlin-3, an Hdm2 antagonist, inhibits tumor adaptation to hypoxia by stimulating the FIH-mediated inactivation of HIF-1 \{alpha\}. Carcinogenesis. 2009;30(10):1768-1775.

75. Chen D, Li M, Luo J, Gu W. Direct interactions between HIF-1alpha and Mdm2 modulate p53 function. J Biol Chem. 2003;278(16): 13595-13598.

76. Yang A, Kaghad M, Caput D, McKeon F. On the shoulders of giants: p63, p73 and the rise of p53. Trends Gene. 2002;18(2):90-95.

77. Lunghi P, Costanzo A, Mazzera L, Rizzoli V, Levrero M, Bonati A. The p53 family protein $\mathrm{p} 73$ provides new insights into cancer chemosensitivity and targeting. Clini Cancer Res. 2009;15(21):6495-6502. 
78. Bergamaschi D, Gasco M, Hiller L, et al. p53 polymorphism influences response in cancer chemotherapy via modulation of p73-dependent apoptosis. Cancer Cell. 2003;3(4):387-402.

79. Stiewe T, Pützer BM. Role of p73 in malignancy: tumor suppressor or oncogene? Cell Death Differ. 2002;9(3):237-245.

80. Zaika AI, Slade N, Erster SH, et al. \{Delta $\}$ Np73, a dominant-negative inhibitor of wild-type p53 and TAp73, is up-regulated in human tumors. J Exp Med. 2002;196(6):765-780.

81. Dominguez G, Garcia JM, Pena C, et al. \{Delta $\}$ TAp73 upregulation correlates with poor prognosis in human tumors: putative in vivo network involving p73 isoforms, p53, and E2F-1. J Clin Oncol. 2006; 24(5):805-815.

82. Rossi M, A ES, Alessandro T, Gerry M, Richard AK. Mechanism of induction of apoptosis by $\mathrm{p} 73$ and its relevance to neuroblastoma biology. Ann N Y Acad Sci. 2004;1028:143-149.

83. Lau LMS, Nugent JK, Zhao X, Irwin MS. HDM2 antagonist nutlin-3 disrupts p73-HDM2 binding and enhances p73 function. Oncogene. 2007;27(17):997-1003.

84. Peirce S, Findley H. The MDM2 antagonist nutlin-3 sensitizes p53-null neuroblastoma cells to doxorubicin via E2F1 and TAp73 Int J Oncology. 2009;34(5):1395-1402.

85. Lau LMS, Wolter JK, Lau JTML, et al. Cyclooxygenase inhibitors differentially modulate p73 isoforms in neuroblastoma. Oncogene. 2009;28(19):2024-2033.

86. Secchiero P, Zerbinati C, Melloni E, et al. The MDM-2 antagonist nutlin-3 promotes the maturation of acute myeloid leukemic blasts. Neoplasia. 2007;9(10):853-861.

87. Becker K, Marchenko N, Maurice M, Moll U. Hyperubiquitylation of wild-type p53 contributes to cytoplasmic sequestration in neuroblastoma. Cell Death Differ. 2007;14(7):1350-1360.
88. Isaacs JS, Saito Si, Neckers LM. Requirement for HDM2 activity in the rapid degradation of p53 in neuroblastoma. J Biol Chem. 2001; 276(21):18497-18506.

89. Slack A, Lozano G, Shohet JM. MDM2 as MYCN transcriptional target: Implications for neuroblastoma pathogenesis. Cancer Letters. 2005;228(1-2):21-27.

90. Slack A, Shohet J. MDM2 as a critical effector of the MYCN oncogene in tumorigenesis. Cell Cycle. 2005;4(7):857-860.

91. Slack AD, Chen Z, Ludwig AD, Hicks J, Shohet JM. MYCN-directed centrosome amplification requires MDM2-mediated suppression of p53 activity in neuroblastoma cells. Cancer Res. 2007;67(6): 2448-2455.

92. Chen L MA, Wood KM, Cole M, et al. p53 is nuclear and functional in both undifferentiated and differentiated neuroblastoma. Cell Cycle. 2007;6(21):2.

93. Van Maerken T, Speleman F, Vermeulen J, et al. Small-molecule MDM2 antagonists as a new therapy concept for neuroblastoma. Cancer Res. 2006;66(19):9646-9655.

94. Michaelis M, Rothweiler F, Klassert D, et al. Reversal of P-glycoproteinmediated multidrug resistance by the murine double minute 2 antagonist nutlin-3. Cancer Res. 2009;69(2):416-421.

95. Van Maerken T, Ferdinande L, Taildeman J, et al. Antitumor activity of the selective MDM2 antagonist nutlin-3 against chemoresistant neuroblastoma with wild-type p53. J Natl Cancer Inst. 2009;101(22):1562-1574.

96. De Laurenzi V, RaschellÃ $;$ G, Barcaroli D, et al. Induction of neuronal differentiation by p73 in a neuroblastoma cell line. J Biol Chem. 2000;275(20):15226-15231.
Cell Health and Cytoskeleton

\section{Publish your work in this journal}

Cell Health and Cytoskeleton is an international, peer-reviewed open access journal focusing on all aspects of cell structure and function contributing to normal physiology and cell health and exploring the pathogenesis of cell dysfunction leading to adverse conditions and disease in the organism. The journal welcomes papers covering original research,

\section{Dovepress}

basic science, reviews and evaluations, guidelines, expert opinion and commentary, case reports and extended reports. The manuscript management system is completely online and includes a very quick and fair peerreview system, which is all easy to use. Visit http://www.dovepress.com/ testimonials.php to read real quotes from published authors. 